\title{
Understanding Phenol Adsorption Mechanisms on Activated Carbons
}

\author{
Leticia F. Velasco, Conchi O. Ania* \\ Instituto Nacional del Carbón, CSIC, P.O. 73, 33080 Oviedo, Spain. \\ * Corresponding author email: conchi@incar.csic.es (CO Ania) \\ Tel.: +34 985118846; Fax +34 985297662
}

\begin{abstract}
The interactions between phenol molecules and activated carbons were investigated in order to understand the adsorption mechanism of this aromatic compound. A series of activated carbons with varied chemical composition but similar porous features were synthesized and submitted to phenol exposure from aqueous phase, followed by thermogravimetric analysis and identification of the desorbed species by temperature programmed desorption coupled with mass spectrometry. Based on these experiments, both physi- and chemisorption sites for phenol were identified on the activated carbons. Our results demonstrate that physisorption of phenol depends strictly on the porosity of the activated carbons, whereas chemisorption depends on the availability of the basal planes in the activated carbons. Thus, oxidation of the carbon can suppress the fraction of chemisorbed phenol since the surface functionalities incorporate to the edges of the basal planes; notwithstanding, hydrophilic carbons may present a small but not negligible contribution of chemisorbed phenol depending on the extent of the functionalization. Moreover, these adsorption sites (chemi-) are recovered by simply removal of the surface functionalities after thermal annealing.
\end{abstract}

Keywords: activated carbon, phenol, physisorption, chemisorption 


\section{Introduction}

Faced with an increasing contamination of water resources, adsorption has become a wellestablished technique to remove pollutants, activated carbon being the prevailing adsorbent for the purification of water with low pollutant concentration. Currently, the loaded carbon is regenerated off-site by heating or steaming in large industrial facilities. Although thermal reactivation is a highly skilled process that ensures that spent carbon is returned to a reusable quality, there is a major issue concerning the economic costs of the reactivation of the spent material. The efficiency of the different regeneration processes of activated carbons largely depends on the following factors: the porous structure of the carbon and the chemical condition of its surface, the physicochemical properties of the adsorbent, the methods applied for regeneration, and the conditions under which the regeneration process is conducted (Dranca et al. 2001; Alvarez et al. 2004; Ania et al. 2005a; Salvador and Sanchez-Jimenez 1996).

Phenol is a widespread persistent organic pollutant commonly present in industrial effluents that has been the subject of a number of investigations, since it is a relatively simple and well characterized molecule. Despite the works devoted to the adsorption, desorption and regeneration of phenol using activated carbons, the interactions between activated carbon surfaces and phenol molecule are still rather unknown and controversial (Radovic et al. 2000; Nesvkaia and GuerreroRuiz 2001; Haydar et al. 2003; Ania et al. 2002; Terzyk 2003).

The aim of this study is to contribute with a novel insight in the understanding of the interactions between phenol molecules and activated carbons with different surface chemistry. Comprehensive knowledge of the adsorption mechanism and identification of the adsorption sites on the carbon surface will contribute to optimize the thermal reactivation process of the carbonaceous adsorbent.

As the first target, we have synthesized carbons with varied surface chemistry by incorporating oxygen functionalities via wet oxidation. Also, the oxidized sample was submitted to a high temperature thermal treatment to remove the created groups; the performance of both samples towards phenol adsorption from aqueous solutions has been investigated and compared with that of the raw material. To identify the nature of phenol adsorption sites (weak interactions or physical adsorption and/or strong interactions or chemisorption), we have characterized the activated carbons before and after phenol exposure by means of different techniques including 
thermogravimetric analysis, temperature programmed desorption coupled to mass spectroscopy, and gas adsorption. The thermal stability of the phenol bonded to each adsorption site has been explored, and coupled to the needs in the thermal reactivation of the exhausted adsorbent. This has allowed us to throw some light into the understanding of the phenol adsorption mechanism on porous carbons, particularly the role of the activated carbon features (porosity and composition).

\section{Experimental}

\subsection{Materials}

A commercial activated carbon -B- obtained from physical activation of coal and supplied by Agrovin SA was used as starting material -particle size fraction of 0.212-0.710 mm-. A second sample with a similar porous structure but different surface chemistry was prepared by wet oxidation at room temperature with a saturated solution of ammonium persulfate in $4 \mathrm{~N}_{2} \mathrm{SO}_{4}(1 \mathrm{~g}$ activated carbon:10 mL of oxidizing agent, and left stirring overnight). After oxidation, the carbon was washed and dried at $110^{\circ} \mathrm{C}$ overnight. The oxidized sample (labeled as $\mathrm{BO}$ ) was further submitted to a thermal treatment at $800{ }^{\circ} \mathrm{C}(\mathrm{BOH})$ under inert atmosphere for 1 hour in order to remove the surface groups and to explore the effect on the chemical, structural and adsorptive features of the activated carbons. Phenol with the highest purity specification was obtained from Aldrich.

\subsection{Textural and chemical characterization of the carbons}

Textural characterization was carried out by measuring the $\mathrm{N}_{2}$ adsorption isotherms at $-196{ }^{\circ} \mathrm{C}$. Before the experiments, the samples were outgassed under vacuum at $120^{\circ} \mathrm{C}$ overnight. The $\mathrm{N}_{2}$ isotherms were used to calculate the specific surface area, $\mathrm{S}_{\mathrm{BET}}$, total pore volume, $\mathrm{V}_{\mathrm{T}}$, and pore size distributions which were evaluated by means of the DFT method using a slit pore geometry. Additionally, the volume of pores smaller than $0.7 \mathrm{~nm}$ (narrow micropores) was assessed from $\mathrm{CO}_{2}$ adsorption isotherms at $0{ }^{\circ} \mathrm{C}$ with the $\mathrm{DR}$ formulism. The materials were further characterized by the determination of the point of zero charge $\left(\mathrm{pH}_{\mathrm{PzC}}\right)$ using a modification of the mass-titration method by Noh and Schwarz (1989). About $0.25 \mathrm{~g}$ of activated carbon was dispersed in a suitable volume of distilled water and stored with constant stirring at room temperature until equilibrium was attained (ca. 48 h). Typical activated carbon/water ratios employed were 1, 2, 4, 6, 8, 10, 12, 14 and 16. Once equilibrium had been reached, the $\mathrm{pH}$ value was measured using a glass electrode. 
Newly distilled water was then added in order to obtain the next solid/weight fraction. The plateau in the plot of equilibrium $\mathrm{pH}$ versus solid weight fraction corresponded to the $\mathrm{pH}_{\mathrm{PZC}}$ value of the activated carbon.

\subsection{Thermal analysis}

Thermal analysis was carried out using a thermogravimetric analyzer from Setaram. Experiments were carried out under an argon flow rate of $50 \mathrm{~cm}^{3} \mathrm{~min}^{-1}$, at a heating rate of $15^{\circ} \mathrm{C} \mathrm{min}{ }^{-1}$, up to a final temperature of $900{ }^{\circ} \mathrm{C}$. For each experiment, about $25 \mathrm{mg}$ of a carbon sample was used. Also, temperature programmed desorption experiments were performed with samples of about $100 \mathrm{mg}$ in a Chemisorb 2750 apparatus from Micromeritics, under the same operating conditions mentioned above. Effluent gases were analyzed by means of a mass quadrupole (Balterzs).

\subsection{Phenol adsorption from solution}

The saturation of the activated carbons was carried out from batch experiments, putting in contact $1 \mathrm{~g}$ of adsorbent with $250 \mathrm{~mL}$ of a solution of the organic compound of known initial concentration. The suspensions were shaken at 500 rpm for 24 hours. Afterwards, the samples were filtered and the adsorbate concentration in the supernatant liquid was measured on a Shimadzu 2501 UV-Vis spectrophotometer at the corresponding wavelength. The exhausted activated carbons were washed with a small amount of water to remove the excess of phenol, dried at $60{ }^{\circ} \mathrm{C}$ for overnight, and kept in dessicator until use. The samples after phenol exposure will be labeled as the name of the corresponding activated carbon followed by "x" (i.e., Bx accounts for saturated B). Some experiments were also carried out by submitting the saturated and dried B carbon (sample Bx) to an outgassing treatment (vacuum + temperature) at 200 and $350{ }^{\circ} \mathrm{C}$. These samples will be labeled as BxoutT, being $\mathrm{T}$ the temperature of the treatment.

\section{RESULTS AND DISCUSSION}

\subsection{Characterization of the activated carbons}

Detailed nanotextural parameters of the studied activated carbons obtained from gas adsorption data $\left(\mathrm{N}_{2}\right.$ and $\mathrm{CO}_{2}$ at $-196{ }^{\circ} \mathrm{C}$ and at $0{ }^{\circ} \mathrm{C}$, respectively) are shown in Table 1 . All the carbons displayed type I nitrogen adsorption isotherms (not shown) according to the BDDT classification (Brunauer et al. 1940), which is characteristic of microporous carbons. It should be noted that the 
oxidation treatment (sample BO) did not significantly alter the porous features of the as-received carbon, since only a small fall in the apparent surface area and pore volumes was observed. Also, the removal of the surface groups from the oxidized carbon does not seem to have a deep impact on the porosity of the annealed $\mathrm{BOH}$ carbon. An exhaustive analysis of the textural properties by means of the $\mathrm{CO}_{2}$ adsorption isotherms revealed that the oxidation of the parent carbon caused a slight increase in the amount of $\mathrm{CO}_{2}$ adsorbed. The $\mathrm{CO}_{2}$ adsorption isotherm of the parent $\mathrm{B}$ carbon was recovered after the thermal annealing at $800{ }^{\circ} \mathrm{C}$ (carbon $\left.\mathrm{BOH}\right)$, suggesting that such effect was likely attributed to the functionalization of the carbon surface.

As expected regarding surface chemistry, the oxidation of the activated carbon caused an increase in the oxygen content accompanied by a sharp fall in the $\mathrm{pH}_{\mathrm{PZC}}$ (Table 2). The mass loss (dry basis) of the raw carbons goes from 2 wt.\% for B carbon to 9.5 wt.\% in sample BO, confirming the incorporation of surface functional groups upon oxidation. Moreover, the DTG profiles of BO carbon show a peak centered at about $250^{\circ} \mathrm{C}$, corresponding to the decomposition of labile (typically acidic) groups. Above $400{ }^{\circ} \mathrm{C}$, a broad desorption band is obtained, assigned to more stable oxygen-containing surface functionalities (Bandosz and Ania 2006). According to expectations, the thermal treatment at $800{ }^{\circ} \mathrm{C}$ of the oxidized carbon caused the complete removal of the surface functionalities (total mass loss below $1 \mathrm{wt} . \%$ ), rendering a hydrophobic carbon (sample BOH).

\subsection{Phenol uptake in activated carbons with varied properties}

The effect of the activated carbons features on phenol adsorption mechanism was investigated using un-buffered aqueous solutions and evaluating the phenol retention capacity on each adsorbent. The adsorption capacities for the investigated carbons are 315, 198 and $382 \mathrm{mg} \mathrm{g}^{-1}$ for $\mathrm{B}, \mathrm{BO}$ and $\mathrm{BOH}$, respectively. It appears that the oxidation of the carbon surface has a large impact on the removal of phenol, which is much larger in the activated carbons of hydrophobic nature (uptake falls by almost $40 \%$ ). Moreover, the removal of the surface functional groups allows to recover the large capacity of the activated carbon.

These values are in good agreement with the trend observed in the corresponding mass losses after phenol exposure evaluated from the thermal analysis (ca. 9, 5 and 11 wt.\% for Bx, BOx and BOHx respectively). This data is also consistent with previous works found in the literature about phenol removal on activated carbons (Moreno-Castilla et al. 1995; Ania et al. 2002; Castillejos-Lopez, et 
al. 2008; Toth et al. 2009; Terzyk, 2003), reporting the negative impact of carbon surface oxidation in the overall phenol uptake.

The adsorption performance on a given porous material is traditionally related to the chemical composition and the porous features of the adsorbents. In this sense, according to the similar textural characteristics of the three studied carbons (Table 1), one would expect similar phenol uptakes, or perhaps a somewhat higher uptake on the oxidized carbon -with the highest volume of narrow micropores-. Thus based on the experimental data obtained, it is clear that phenol retention cannot be exclusively attributed to the porosity and it should rather be discussed in terms of the different surface chemistry of the studied activated carbons.

\subsection{Thermal analysis of the activated carbons after phenol exposure}

For this reason we have investigated the samples after phenol exposure by means of thermogravimetric analysis and temperature programmed desorption coupled to mass spectrometry (TPD-MS). The corresponding weight derivatives of the thermal analysis profiles (DTG) as well as some of the mass fragments detected by TPD-MS are shown in Figures 1 and 2. The most important fragments detected between 25 and $900{ }^{\circ} \mathrm{C}$ and their assignments were $\mathrm{CH}_{3}{ }^{+}(\mathrm{m} / \mathrm{z}$ 15), $\mathrm{H}_{2} \mathrm{O}(\mathrm{m} / \mathrm{z} 18), \mathrm{CO}(\mathrm{m} / \mathrm{z} 28), \mathrm{O}_{2}(\mathrm{~m} / \mathrm{z} 32), \mathrm{CO}_{2}(\mathrm{~m} / \mathrm{z} 44), \mathrm{C}_{5} \mathrm{H}_{5}(\mathrm{~m} / \mathrm{z} 66), \mathrm{C}_{5} \mathrm{H}_{11}(\mathrm{~m} / \mathrm{z} 78), \mathrm{C}_{6} \mathrm{H}_{5} \mathrm{O}$ (m/z 93), $\mathrm{C}_{6} \mathrm{H}_{6} \mathrm{O}(\mathrm{m} / \mathrm{z} 94)$ and $\mathrm{C}_{12} \mathrm{H}_{8} \mathrm{O}(\mathrm{m} / \mathrm{z}$ 168). For clarity purposes, only those fragments corresponding to $\mathrm{m} / \mathrm{z} 18,66,78$ and 94 are plotted since they provide most useful information about phenol adsorption mechanisms and thermal cracking.

The DTG profiles after phenol exposure allow the different adsorption sites on the studied carbons to be identified (Figure 1). Three well defined desorption peaks and two wide humps at temperatures above $500^{\circ} \mathrm{C}$ can be distinguished in $\mathrm{B}$ and $\mathrm{BOH}$ samples, as opposed to the oxidized carbon with only two peaks. The first peak at around $100{ }^{\circ} \mathrm{C}$ is typically assigned to moisture retained in the porous network of the carbons since phenol exposure is carried out from an aqueous solution. This assignment was confirmed by the m/z 18 signal detected by TPD-MS (Figure 2).

The peaks at higher temperatures are attributed to phenol retention on different sites of adsorption since they are only detected in the saturated carbons, with the exception of the high temperature humps in BO carbon due to the decomposition of the surface functional groups (Figure 1). It is generally accepted that the fraction of phenol adsorbed at low temperatures corresponds to 
physisorption whereas the peaks at higher temperatures belong to chemisorbed species (MorenoCastilla et al. 1995; Ania et al. 2005b; Castillejos-Lopez et al. 2008; Toth et al. 2009). In good agreement with previous works and based on DTG data it would seem that phenol is both physiand chemisorbed in the hydrophobic B and BOH carbons, whereas chemisorption is not detected in the oxidized carbon. However, a deep analysis of the mass to charge fragments by TPD-MS revealed interesting and surprising findings.

An important observation is that when the raw carbon is oxidized the temperature of the first desorption band observed in DTG profiles (physisorbed phenol) does not change. In fact, it remains somewhat the same for the three investigated carbons, which is consistent with their similar textural properties. This result indicates that the presence of the oxygen functional groups at the pores entrance does not hamper the physisorption of phenol. Furthermore, when the functional groups are removed from the carbon surface (sample BOH) the desorption peak still remains in the same position -as the porosity of the carbon remains unaltered after the thermal treatment-. At converse, upon removal of the surface functionalities, the high temperature phenol desorption peaks reappear -those detected in the hydrophobic B carbon but not after oxidation-. This result also suggests that there does not exist specific interactions between phenol molecules and the oxygen functionalities (ca. H-bonding and/or anchoring through the $\mathrm{CO}$ moiety in carboxylic acids on the carbon surface), which has been a controversial issue in the literature so far (Nesvkaia and Guerrrero-Ruiz 2001; Haydar et al. 2003).

Following the evolution of the different species throughout the whole temperature desorption range (Figure 2) it can be seen that phenol is retained on different surface sites. According to literature (Smith 2004), fragmentation of phenol due to ionization in the MS spectrometer yields the fragments $94\left(\mathrm{C}_{6} \mathrm{H}_{5} \mathrm{O}^{+}\right)$and $66\left(\mathrm{C}_{5} \mathrm{H}_{5}{ }^{+}\right)$, with $99 \%$ relative abundance for $\mathrm{m} / \mathrm{z} 94$. During the TPD experiments both fragments appear at the same temperature, thus confirming their formation due to MS cracking of phenol. Additionally, various $\mathrm{m} / \mathrm{z}$ signals were also observed at temperatures above $500{ }^{\circ} \mathrm{C}$ in the profiles of all the saturated carbons (i.e., $\mathrm{m} / \mathrm{z} 78$ assigned to $\mathrm{C}_{6} \mathrm{H}_{6}{ }^{+}$). These are due to the thermal cracking of phenol above this temperature (Spielmann and Cramers 1972; Cypres and Betfens 1974), which generates benzene, naphthalene, dibenzofuran, $\mathrm{H}_{2} \mathrm{O}, \mathrm{CO}, \mathrm{H}_{2}$ and $\mathrm{CH}_{4}$, along with a solid residue as main sub-products. Consequently, the intensity of the $\mathrm{m} / \mathrm{z} 94$ signal is expected to decrease above $500^{\circ} \mathrm{C}$ at expenses of an increase in the mass to charge signals of phenol cracking sub-products. 
According to this, the appearance of the $\mathrm{m} / \mathrm{z} 78\left(\mathrm{C}_{6} \mathrm{H}_{6}{ }^{+}\right)$signal along with an increase in the m/z 15, 28 and 44 -assigned to $\mathrm{CH}_{4}$, $\mathrm{CO}$ and $\mathrm{CO}_{2}$, respectively- in the profiles of all the preadsorbed carbons (even in the oxidized sample) has to be linked to the occurrence of high temperature phenol desorption sites. Moreover, the temperature of the $\mathrm{m} / \mathrm{z} 78$ peak in carbons B and $\mathrm{BOH}$ matches with the desorption humps observed in the DTG profiles (Figure 1) at high temperatures.

It should also be highlighted that several m/z 18 signals are observed for all the studied carbons, with broad bands which maxima temperature matches with the evolution of the $\mathrm{m} / \mathrm{z}$ 94, 78 signals. Similar water desorption profiles have been reported for the adsorption of aromatics from aqueous solution (Nesvkaia and Guerrrero-Ruiz 2001; Castillejos-Lopez et al. 2008), and attributed this finding to the desorption of water associated with the adsorbate in supramolecules. However, one should also take in mind that water is also originated from the thermal cracking of phenol molecules: above $700{ }^{\circ} \mathrm{C}$ phenol thermal degradation yields between 6-30 wt.\% water (Cypres and Betfens 1974). This would also explain the small hump observed in the $\mathrm{m} / \mathrm{z} 18$ profiles at temperatures above $700{ }^{\circ} \mathrm{C}$. Another factor to consider is that water may also appear in condensation reactions of phenol to render dibenzofuran (around $400-500^{\circ} \mathrm{C}$ ). For instance, we have detected the $\mathrm{m} / \mathrm{z} 168\left(\mathrm{C}_{12} \mathrm{H}_{8} \mathrm{O}\right)$ signal corresponding to this compound in the desorption profiles of $\mathrm{B}$ and $\mathrm{BOH}$. Thus, it may be inferred that water desorption between $200-500{ }^{\circ} \mathrm{C}$ may be linked to the water molecules associated to phenol during the adsorption process, while at higher temperatures the contribution due to phenol thermal cracking cannot be neglected.

Comparatively, the nature and number of phenol adsorption sites change with the nature of the activated carbons. For the hydrophobic B and BOH carbons, phenol seems to be adsorbed through physisorption and multiple chemisorption sites, whereas the chemisorption appears to be strongly reduced after oxidation. To throw some light on this issue, we have analyzed saturated B carbon after outgassing at 200 and $350{ }^{\circ} \mathrm{C}$ during 3 hours and overnight, respectively.

The DTG and TPD-MS profiles of samples Bxout200 and Bxout350 are shown in Figures 3 and 4, respectively. Outgassing at $200{ }^{\circ} \mathrm{C}$ (sample Bxout200) provoked almost the complete removal of the fraction of physisorbed phenol (Figure 3), while the chemisorbed fraction remained unaltered: the three bands at temperatures above $300{ }^{\circ} \mathrm{C}$ are still detected, as well as the corresponding $\mathrm{m} / \mathrm{z}$ 94 and 78 signals (the shape of the TPD-MS profiles above $300{ }^{\circ} \mathrm{C}$ was not modified with respect 
to Bx). Analogously, after outgassing at $350{ }^{\circ} \mathrm{C}$ only the $\mathrm{m} / \mathrm{z} 94,78$ signals at $500{ }^{\circ} \mathrm{C}$ and above are observed, indicating the complete removal of physisorbed phenol as well as a fraction of chemisorbed phenol. It is noteworthy that compared to the removal of the physisorbed fraction (outgassing at $200{ }^{\circ} \mathrm{C}$ for 3 hours), not only a higher temperature but also a much larger time of the outgassing treatment is required to achieve a partial removal of the chemisorbed phenol from the surface of the activated carbon (outgassing at $350^{\circ} \mathrm{C}$ for 17 hours). This performance highlights the differences in the nature of the forces involved in physical and chemical adsorption, being the latest considerably stronger.

The removal of a fraction of adsorbed phenol is also confirmed by the textural characterization of Bxout200 and Bxout350 (Table 1); after the outgassing treatment there is an increase in the surface area and micropore volumes, being more notable in the sample treated at $350{ }^{\circ} \mathrm{C}$. The outgassing treatment of the saturated samples assures that all the evolved molecules are withdrawn from the carbon surface preventing any deposition; this demonstrates that the high temperature adsorption sites observed in the outgassed series correspond to phenol uptake from solution, and not to reorganization of the desorbed phenol molecules during the thermal treatment, nor to its degradation sub-products on the porosity of the carbons.

What it's interesting to note is that the outgassing treatment provokes changes in the water desorption profiles, following a similar trend than that observed for signal $\mathrm{m} / \mathrm{z} 94$. This confirms that water desorption below $400{ }^{\circ} \mathrm{C}$-where phenol thermal degradation is discarded- is associated to phenol molecules retained on the activated carbon surfaces, as suggested in the literature (Nesvkaia and Guerrrero-Ruiz 2001).

After oxidation of the pristine activated carbon, the peak corresponding to physisorbed phenol does not change as it was mentioned above (Figure 1). This was also clearly observed in Figure 2; however, small peaks in the m/z 94 (Figure 2, inset) and m/z 78 were also detected at moderate temperatures between $400-600^{\circ} \mathrm{C}$. Despite the intensity of these signals is much lower than in the case of hydrophobic carbon, this demonstrates that there still remains a small but non negligible contribution of chemisorbed phenol in the oxidized carbon. It would seem then that only a part of the chemisorption sites is blocked during the oxidation. In agreement with this finding, the $\mathrm{m} / \mathrm{z} 18$ hump above $500{ }^{\circ} \mathrm{C}$ is observed for BO carbon (not detected in its corresponding blank in water), 
confirming either water associated to phenol molecules or water released during phenol thermal cracking.

The negative impact of carbon oxidation on the chemisorption of phenol has been recently reported (Toth et al. 2009), although the authors state that physisorption of phenol is favoured in hydrophilic carbons. However, our results demonstrate that physisorption does no depend on the oxidation state of the carbon material but on its porosity. In fact, the mass loss in the saturated carbons accounting for the physisorbed fraction of phenol is 5 wt.\% for all the carbons. Only chemisorptions is affected (largely suppressed) by the presence of oxygen-containing groups. In any case, both findings contrast with previous works reporting one single m/z 94 peak in oxidized carbons, where it is concluded that oxygen-containing surface functional groups inhibit the phenol chemisorption on activated carbons (Castillejos-Lopez et al. 2008).

To understand this issue one should take into account that we have carried out a mild oxidation -in order to avoid drastic changes in the porosity of the carbons-, and that the oxygen content in BO carbon is not very large (Table 2). Since the surface functionalities are mainly incorporated at the edges of the basal planes (Donnet 1982), it seems that they have not been fully decorated with the functional groups; thus there would still remain some non-blocked chemisorption sites on the oxidized carbon. This would explain the differences observed with previous works reported in the literature, where unfortunately details on the oxidation extent on the carbons are missing (Castillejos-Lopez et al. 2008).

Moreover, the DTG and TPD-MS of carbon BOH (Figures 1 and 2) confirm this premise; the high temperature desorption peaks in the DTG profiles reappear in BOH carbon, while the intensity of the $\mathrm{m} / \mathrm{z} 94$ and 78 signals above $500^{\circ} \mathrm{C}$-which were partially removed in the oxidized carbonincreases significantly. In fact, the profiles of carbon BOH are similar to those of the as-received B carbon, which demonstrates that the sites for phenol chemisorption are restored by removal of the oxygen functionalities created upon oxidation.

On the other hand, the complete removal of phenol is not achieved even if high temperatures (ie., $800{ }^{\circ} \mathrm{C}$ ) are applied; despite the desorption profiles do not reveal the presence of any peak after the high temperature treatment, the porosity of the activated carbons is not fully recovered (ca. $33 \%$ fall in surface area and pore volumes after treatment at 700 and $800^{\circ} \mathrm{C}$, respectively). The reason for this is that thermal cracking of phenol gives rise to a solid residue (yield between 30 wt.\%) 
which deposits on the carbon surface and causes pore plugging on the activated carbon, thus limits the thermal regeneration of the exhausted materials (Ania et al. 2005a; Cypres and Betfens 1974). In other words, if regeneration of exhausted activated carbons is carried out at moderate temperatures, there is a fraction of chemisorbed phenol that is not eliminated. In contrast, higher temperatures also provoke a collapse in the porous features of the activated carbons, although as a consequence of the thermal cracking of the phenol molecules forming coke deposits.

Summarizing, it may be concluded then that DTG coupled to TPD-MS allow to attribute the desorption peaks to different phenol adsorption sites: physisorbed phenol (ca., $200{ }^{\circ} \mathrm{C}$ ), and three chemisorption sites (ca., 400, 550, $700^{\circ} \mathrm{C}$ ). This also confirms that physisorption of phenol does not depend on the oxidation state of the activated carbons but on their porosity. In contrast, chemisorption seems to be favoured in hydrophobic carbons due to interactions of phenol molecules with the edges of the graphene planes in the carbon. Consequently, chemisorbed phenol may be suppressed when these edges are decorated with the surface functionalities, the extension of which depends on the coverage/blocking of the chemisorption sites.

\section{CONCLUSIONS}

This work investigates the mechanisms of phenol adsorption on activated carbons by means of thermal analysis of the adsorbents after phenol exposure, with the identification of the desorbed species by temperature programmed desorption coupled with mass spectrometry. A series of activated carbons with similar textural properties and different surface chemistry were investigated; these were obtained from a hydrophobic carbon that was oxidized to create oxygencontaining functional groups, and subsequently annealed to remove them. The results demonstrate that physisorption of phenol depends strictly on the porosity of the activated carbons, regardless the oxidation state of the carbon surface. Besides physisorption, phenol molecules interact with the edges of the basal planes in the carbons, showing various chemisorption sites desorbing at temperatures above $400{ }^{\circ} \mathrm{C}$ (likely due to different orientations of phenol molecules). Consequently, the incorporation of the surface functionalities on the edges of the basal planes -via carbon oxidation- blocks the chemisorption sites reducing the interactions. However, hydrophilic carbons may present a small but not negligible contribution of chemisorbed phenol depending on the extent of the functionalization. 


\section{Acknowledgements}

This work was supported by the Spanish Ministry of Science and Innovation, MICINN (project CTM2008-01956) and CSIC (200980I131). LFV is grateful to the Spanish Council for Scientific Research (CSIC) for her JAE predoctoral fellowship.

\section{REFERENCES}

Alvarez, P.M., Beltran, F.J., Gomez-Serrano, V., Jaramillo, J., Rodríguez, E.M.: Comparison between thermal and ozone regenerations of spent activated carbon exhausted with phenol. Water Res. 38, 2155-2165 (2004)

Ania, C.O., Menendez, J.A., Parra, J.B., Pis, J.J.: Effect of microwave and conventional regeneration on the microporous and mesoporous network and on the adsorptive capacity of activated carbons, Micropor. Mesopor. Mat. 85, 7-15 (2005a).

Ania, C.O., Parra, J.B., Pevida, C., Arenillas, A., Rubiera, F., Pis, J.J.: Pyrolysis of activated carbons exhausted with organic compounds. J. Anal. Appl. Pyrol. 74, 518-24 (2005b)

Ania, C.O., Parra, J.B., Pis, J.J.: Influence of surface chemistry on active carbon adsorption of selected organic compounds. Fuel Process. Tech., 77, 264-271 (2002)

Bandosz, T.J., Ania, C.O.: Surface chemistry of activated carbons and its characterization. In T.J., Bandosz (ed.), Activated Carbon Surfaces in Environmental Remediation, in the series Interface Science and Technology, vol. 7, pp. 159-229. Elsevier, New York, 2006.

Brunauer, S., Deming, L.S., Deming, W.E., Teller, E.: On a theory of the van der Waals adsorption of gases, J. Am. Chem. Soc. 62, 1723-1732 (1940).

Castillejos-Lopez, E., Nevskaia, D.M., Munoz, V., Guerrero-Ruiz, A.: On the interactions of phenol, aniline and p-nitrophenol on activated carbon surfaces as detected by TPD. Carbon, 46, 870-875 (2008)

Cypres, R., Betfens, B.: Mecanismes de fragmentation pyrolytique du phenol et des cresols, Tetrahedron, 30, 1253-1260 (1974)

Donnet, J.B.: Structure and reactivity of carbons: from carbon black to carbon composites. Carbon 20, 267-282 (1982)

Dranca, Y., Lupascu, T., Vogelsang, K., Monahova, L.: Utilization of thermal analysis to establish the optimal conditions for regeneration of activated carbons, J. Therm. Anal. Cal. 64, 945-953 (2001)

Haydar, S., Ferro-Garcia, M.A., Rivera-Utrilla, J., Joly, J.P.: Adsorption of p-nitrophenol on an activated carbon with different oxidations, Carbon 41, 387-395 (2003)

Moreno-Castilla, C., Rivera-Utrilla, J., Joly, J.P., Lopez-Ramon, M.V., Ferro-Garcia, M.A., Carrasco-Marin, F.: Thermal regeneration of an activated carbon exhausted with different substituted phenols. Carbon 33, 1417-1423 (1995)

Nevskaia, D.M., Guerrero-Ruiz, A.: Comparative study of the adsorption from aqueous solutions and the desorption of phenol and nonylphenol substrates on activated carbons. J. Colloid Interf.

Sci. 234, 316-321 (2001) 
Noh, J.S., Schwarz, J.A.: Estimation of the point of zero charge of simple oxides by mass titration. J. Colloid Interface Sci. 130, 157-164 (1989)

Radovic, L.R., Moreno-Castilla, C., Rivera-Utrilla, J.: Carbon materials as adsorbents in aqueous solutions. In: Radovic, L.R. (ed). Chemistry and physics of carbon, vol. 27, p. 227-405, Marcel Dekker, 2000.

Salvador, F., Sanchez-Jimenez, C.: A new method for regenerating activated carbon by thermal desorption with liquid water under subcritical conditions. Carbon 34, 511-516 (1996)

Smith, M.R. In Understanding Mass Spectra: A Basic Approach. p. 131. Wiley \& Sons, Inc. New Jersey, 2004.

Spielmann, R., Cramers, C.A.: Cyclopentadienic Compounds as Intermediates in the Thermal Degradation of Phenols. Kinetics of the Thermal Decomposition of Cyclopentadiene, Chromatographia 5, 295-300 (1972)

Terzyk, A.P.: Further insights into the role of carbon surface functionalities in the mechanism phenol adsorption J. Colloid Interf. Sci. 268, 301-329 (2003)

Toth, A., Novak, C., Laszlo, K.: The effect of ionic environment on the TG response of phenol loaded PET-based porous carbons, J. Therm. Anal. Calorim., 97, 273-280 (2009) 
Figure 1. Desorption profiles (DTG) before and after phenol exposure of the asreceived carbons

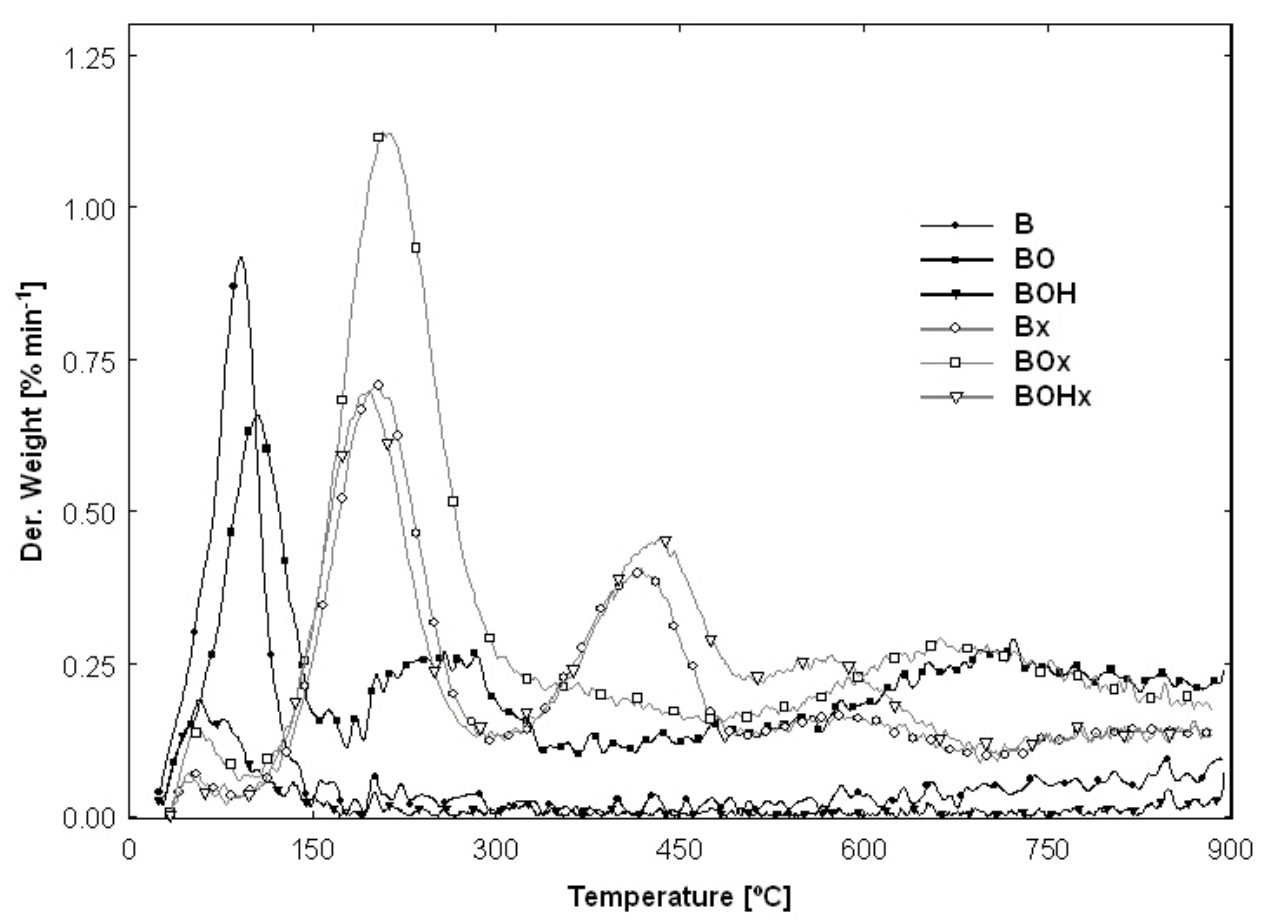



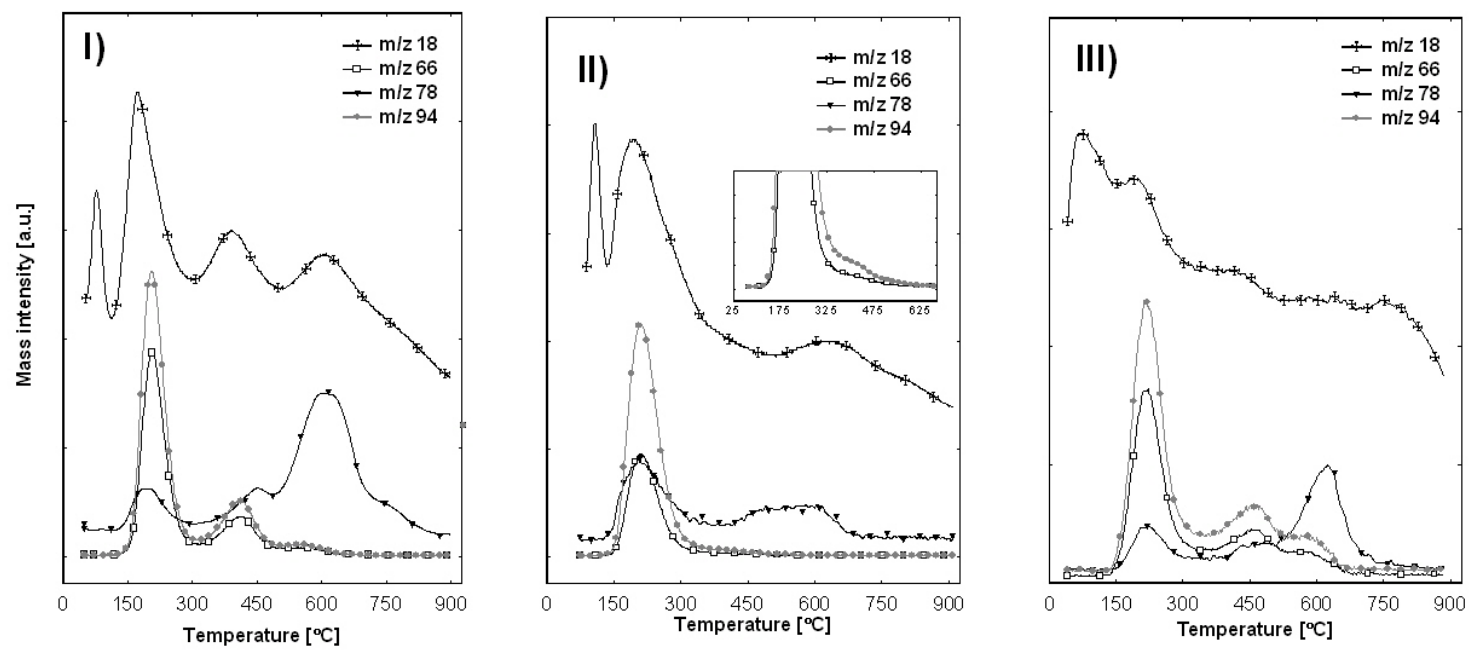

Figure 2. TPD-MS profiles after phenol exposure of i) carbon B, ii) carbon $\mathrm{BO}$ and iii) carbon BOH. Inset: magnification of the $\mathrm{m} / \mathrm{z}$ 66, 94 signals. 


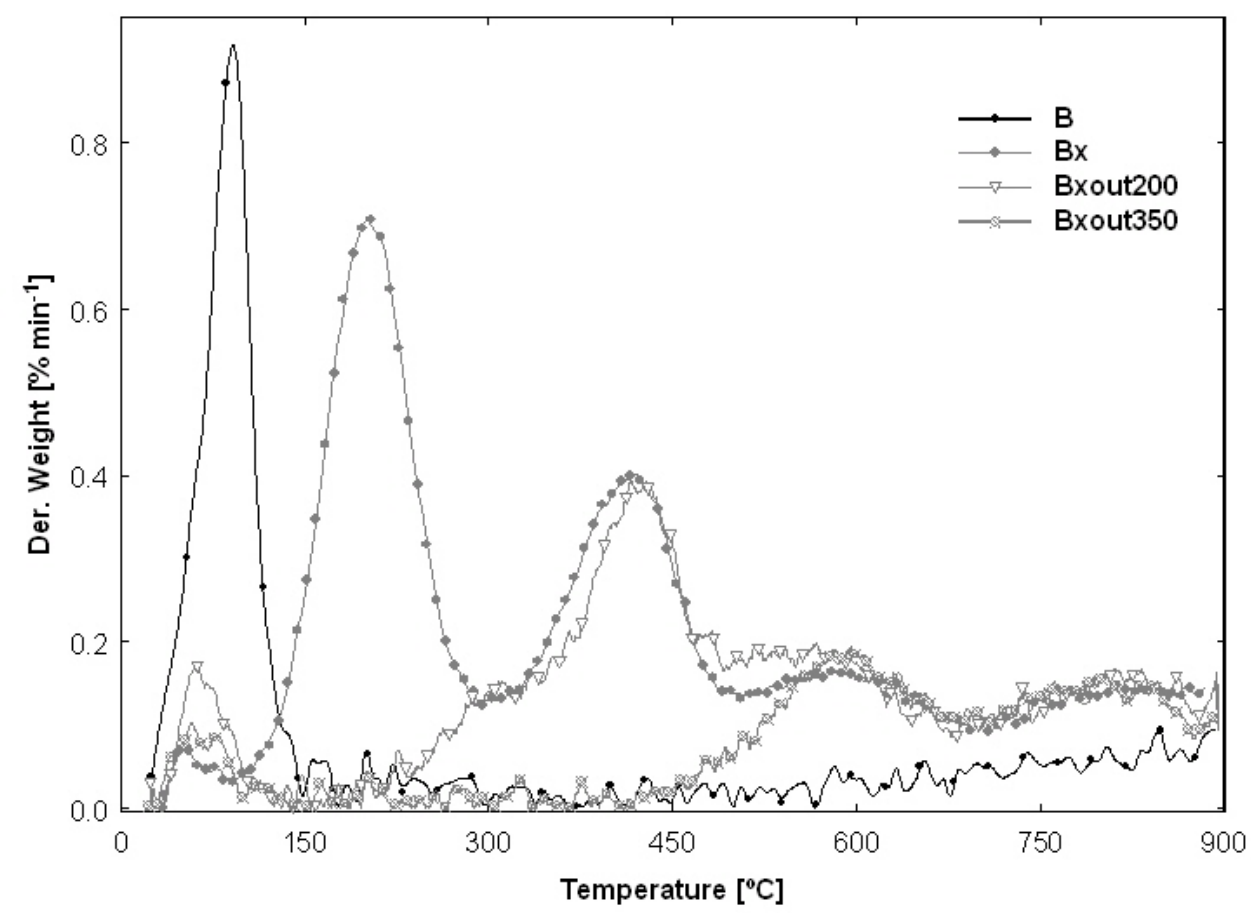

Figure 3. Desorption profiles (DTG) before and after phenol exposure of saturated carbon B after outgassing at different temperatures. 
Figure 4. TPD-MS profiles after phenol exposure of the saturated B carbon after i) outgassing at $200^{\circ} \mathrm{C}$ and ii) outgassing at $350^{\circ} \mathrm{C}$.
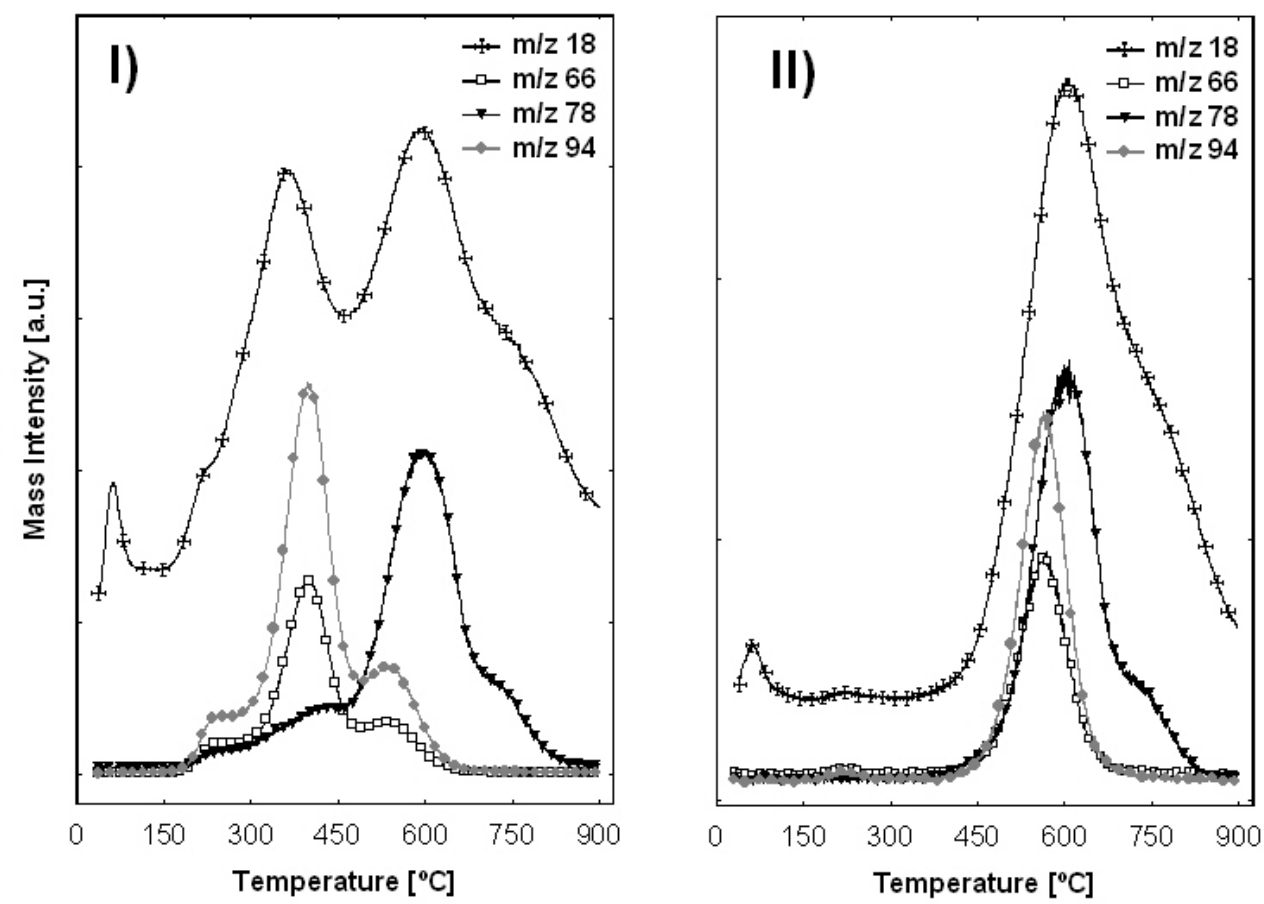
Table 1. Main textural parameters determined from gas adsorption data of the pristine (B), oxidized (BO) and annealed carbon (BOH), before and after phenol exposure (series $\mathrm{x}$ ), and after outgassing at different temperatures (series out).

\begin{tabular}{|c|c|c|c|c|c|c|}
\hline Sample & $\begin{array}{c}S_{\text {BET }} \\
{\left[\mathrm{m}^{2} \mathrm{~g}^{-1}\right]}\end{array}$ & $\begin{array}{l}V_{\text {TOTAL }^{a}} \\
{\left[\mathrm{~cm}^{3} \mathbf{g}^{-1}\right]}\end{array}$ & $\begin{array}{c}\mathrm{V}_{\text {MICROPORES }}{ }^{\mathrm{b}} \\
{\left[\mathrm{cm}^{3} \mathrm{~g}^{-1}\right]}\end{array}$ & $\begin{array}{c}\text { V MESOPORES }^{b} \\
{\left[\mathrm{~cm}^{3} \mathrm{~g}^{-1}\right]}\end{array}$ & $\begin{array}{c}\mathrm{W}_{0, \mathrm{~N} 2}{ }^{\mathrm{c}} \\
{\left[\mathrm{cm}^{3} \mathrm{~g}^{-1}\right]}\end{array}$ & $\begin{array}{l}W_{0, \mathrm{CO} 2}{ }^{\mathrm{c}} \\
{\left[\mathrm{cm}^{3} \mathrm{~g}^{-1}\right]}\end{array}$ \\
\hline B & 1031 & 0.524 & 0.316 & 0.089 & 0.470 & 0.190 \\
\hline BO & 989 & 0.501 & 0.306 & 0.073 & 0.470 & 0.233 \\
\hline ВOH & 1045 & 0.524 & 0.319 & 0.088 & 0.460 & 0.188 \\
\hline $\mathbf{B x}$ & 404 & 0.223 & 0.113 & 0.047 & 0.130 & 0.057 \\
\hline Bxout200 & 539 & 0.289 & 0.153 & 0.060 & 0.235 & 0.072 \\
\hline Bxout350 & 597 & 0.318 & 0.172 & 0.064 & 0.243 & 0.121 \\
\hline
\end{tabular}

Table 2. Ultimate analysis and $\mathrm{pH}$ of the point of zero charge of the studied carbons.

\begin{tabular}{lcccccc}
\hline \multicolumn{4}{c}{ Ultimate analysis (wt. \% dry basis) } & \multicolumn{1}{c}{ pH $_{\text {PzC }}$} \\
\cline { 1 - 5 } & C & H & N & S & O & \\
\hline B & 96.3 & 0.6 & 0.7 & 0.3 & 2.1 & 8.9 \\
BO & 90.7 & 0.7 & 0.7 & 0.4 & 7.5 & 3.3 \\
BOH & 97.7 & 0.4 & 0.1 & 0.4 & 1.4 & 6.6 \\
\hline
\end{tabular}

